\title{
COURRIER
}

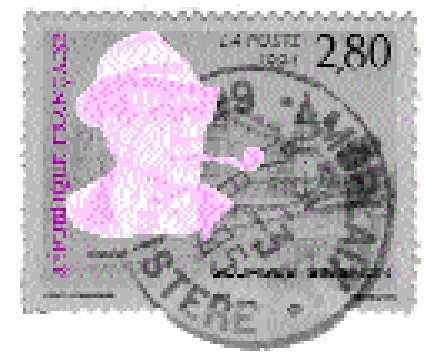

\section{La glycosylation module aussi la fonction des oncogènes}

A propos de l'article de Bernard Vandenbunder paru dans le numéro d'octobre 1995 de médecine/sciences ( ${ }^{\circ} 10$, vol. 11, p. 1465), parmi les propositions pour la période à venir, il était mentionné la nécessité de «chercher à comprendre quel peut être le rôle de la phosphorylation d'un facteur de transcription dans la prolifération ou la différenciation cellulaire».

Compte tenu de l'importance reconnue des glycanes et de la glycosylation dans le domaine de la santé (contrôle des réponses immunitaires et inflammatoires, phénomènes de cancérisation, formation de métastases...) il est nécessaire de mentionner aussi la glycosylation comme modulateur de la fonction de facteurs de transcription comme Sp1, ou d'oncoprotéines telles que c-Myc comme cela a été montré récemment. En effet, il y aurait compétition entre phosphorylation et glycosylation pour moduler la fonction de l'oncoprotéine c-Myc. Cela a été montré par l'équipe de
Gary Hart [Glycosylation of the cmyc transactivation domain. Proc Natl Acad Sci USA 1995; 92 : 441721].

Ces auteurs montrent tout d'abord que l'oncoprotéine c-Myc est O-glycosylée. Puis en utilisant des mutants de délétion, ils montrent que les sites de O-glycosylation $(\mathrm{O}-$ GlcNAc) sont situés près ou dans le domaine de transactivation (TAD), et que ces sites sont distants des sites majeurs de phosphorylation catalysés par la caséine kinase II.

Ce type de glycosylation est dynamique, puisqu'il y aurait une relation réciproque entre glycosylation et phosphorylation de c-Myc, ce qui modulerait sa fonction

\section{Michèle Aubery}

Inserm U. 180, 45, rue des Saints-Pères, 75006 Paris, France.

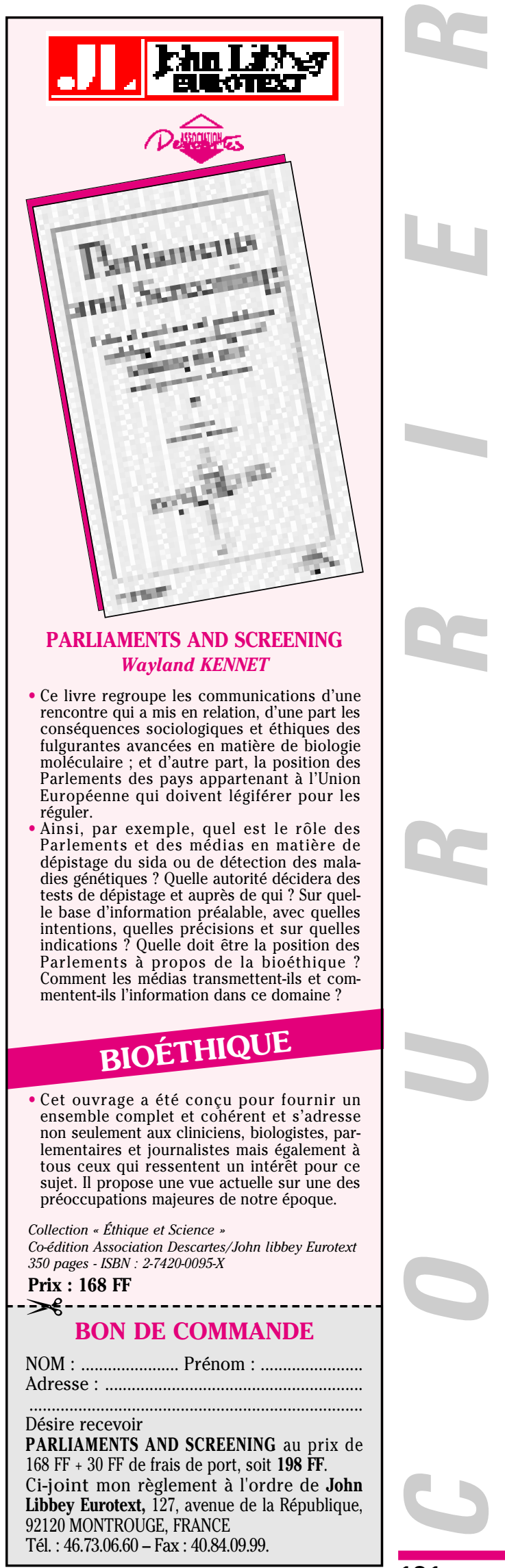

